\title{
On Minimum-B Stabilization of Electrostatic Drift Instabilities*
}

\author{
R. SAISon and H. K. Wimmel \\ Institut für Plasmaphysik GmbH, Garching bei München \\ (Z. Naturforschg. 21 a, 1953-1959 [1966] ; received 1 July 1966)
}

\begin{abstract}
A check is made of a stabilization theorem of Rosenbluth and Krall (Phys. Fluids 8, 1004 [1965]) according to which an inhomogeneous plasma in a minimum- $B$ field $(\beta \ll 1)$ should be stable with respect to electrostatic drift instabilities when the particle distribution functions satisfy a condition given by $\mathrm{T}_{\mathrm{AYlo}}$, i. e. when $f_{0}=f(W, \mu)$ and $\partial f / \partial W<0$. Although the dispersion relation of Rosenbluth and Krall is confirmed to first order in the gyroradii and in $\varepsilon \equiv \mathrm{d} \ln B / \mathrm{d} x$ the stabilization theorem is refuted, as also is the validity of the stability criterion used by RosENBLUTH and KraLL, $\langle\boldsymbol{j} \cdot \boldsymbol{E}\rangle \geqq 0$ for all real $\omega$. In the case $\omega_{\text {pi }} \gg\left|\Omega_{\mathrm{i}}\right|$ equilibria are given which satisfy the condition of $\mathrm{T}_{\mathrm{AYLO}}$ and are nevertheless unstable. For instability it is necessary to have a non-monotonic $v \perp$ distribution; the instabilities involved are thus loss-cone unstable drift waves. In the spatially homogeneous limiting case the instability persists as a pure loss cone instability with $\operatorname{Re}[\omega]=0$. A necessary and sufficient condition for stability is $D(\omega=\infty, k, \ldots) \leq k^{2}$ for all $k$, the dispersion relation being written in the form $D(\omega, k, K, \ldots)=k^{2}+K^{2}$. In the case $\omega_{\text {pi }} \ll\left|\Omega_{\mathrm{i}}\right|$ adherence to the condition given by $\mathrm{T}_{\mathrm{AYLOR}}$ guarantees stability.
\end{abstract}

A stabilization theorem for electrostatic drift instabilities has been published by Rosenbluth and $\mathrm{K}_{\text {RALL }}{ }^{1}$. This states that an inhomogeneous plasma in a minimum- $B$ field with $\beta \ll 1$ should be stable with respect to electrostatic drift instabilities when the distribution functions of the ions and electron satisfy a condition given by $\mathrm{T}_{\mathrm{AYLOR}}{ }^{2}$. This condition stipulates that the distribution functions in equilibrium should depend only on the particle energy and magnetic moment and that the energy distribution be monotonic, that is $f_{0}=f(W, \mu)$ and $\partial f / \partial W$ $<0$.

Since it seemed to us that there were one or two gaps in the proof of Reference 1 we made a check of the result given there. Our calculation takes into account terms of the zeroth and first orders in $\varepsilon \equiv \mathrm{d} \ln B / \mathrm{d} x$ and in the gyroradii. We then obtain the same dispersion relation as in Ref. 1 because the first-order correction terms cancel out. The dispersion relation is of the form $D(\omega, k, K, \ldots)=k^{2}+K^{2}$. The stabilization theorem, on the other hand, is refuted, as also is the applicability of the stability criterion used in ref. ${ }^{1}\langle\boldsymbol{j} \cdot \boldsymbol{E}\rangle \propto \operatorname{Im}[\omega D] \geqq 0$ for all real $\omega$. For equilibria satisfying the TAYLOR condition the Nyquist diagram gives a necessary and sufficient condition for stability, $D(\omega=\infty, k, \ldots) \leqq k^{2}$ for all $k$. In the case $\omega_{\mathrm{pi}} \ll\left|\Omega_{\mathrm{i}}\right|$ it is shown that

\footnotetext{
* Performed under the terms of the agreement between the Institut für Plasmaphysik $\mathrm{GmbH}$ and EURATOM to conduct joint research in the field of plasma physics. - An account of the main results was given in a short paper presented at the Spring Conference of the Fachausschuß „Gas-
}

adherence to the TAYLOR condition does in fact lead to stability. In the reverse limiting case, $\omega_{\mathrm{pi}} \gg\left|\Omega_{\mathrm{i}}\right|$, equilibria can be given which satisfy the TAYLOR condition and are nevertheless unstable. For instability it is necessary to have a non-monotonic $v_{\perp}$-distribution which is automatically realized in minimum- $B$ fields. The instabilities involved are thus loss-cone unstable drift waves. In the spatially homogeneous limiting case, $\varepsilon=0$, a pure loss cone instability with $\operatorname{Re}[\omega]=0$ results.

\section{Magnetic Field}

As in Ref. 1, only a small section of a minimum- $B$ field is studied in which the lines of force can be approximated by straight lines parallel to the $z$-axis of a Cartesian coordinate system. If it is assumed that the equilibrium perturbations under investigation have many wavelengths in this section, one is justified in letting the spatial dimensions of this simplified magnetic field tend to infinity. In particular, $\boldsymbol{B}$ is et equal to $B_{z}(0)(1+\varepsilon x) \boldsymbol{i}_{z}$ with $\varepsilon \neq 0$, and the vicinity of $x=0$ is considered. Even with restriction to the case $\beta \equiv 8 \pi p / B^{2} \ll 1, B$ is not a vacuum field for $\varepsilon \neq 0$ because of its special form. However, the Maxwell equation $c \operatorname{rot} B=4 \pi j$ is

entladungs- und Plasmaphysik" of the Deutsche Physikalische Gesellschaft held in Kiel from 26th to 29th April 1966. 1 M. N. Rosenbluth and N. A. Krall, Phys. Fluids 8, 1004 [1965].

2 J. B. TAYLOR, Phys. Fluids 6, 1529 [1963]. 
not discussed in Ref. 1 . We do not discuss the selfconsistency of the equilibrium configuration either.

The fact that a minimum- $B$ field is involved is no longer directly evident because of the simplified form of $\boldsymbol{B}$. This emerges, however, from the special choice of the distribution functions, $f_{0}=f(W, \mu)$, this being compatible with the assumption of a finite plasma volume only in a minimum- $B$ field.

\section{Equilibrium}

The equilibrium values are all assumed to be independent of $y$ and $z$ and dependent only of $x$. We shall study a collisionless plasma without static electric fields. The distribution functions of the ions and electrons obey the time-independent Vlasov equation:

$$
v_{x} \frac{\partial f_{0}}{\partial x}+\frac{e}{m}(\boldsymbol{v} \times \mathbf{B}) \cdot \frac{\partial f_{0}}{\partial \boldsymbol{v}}=0 ; \quad e \lessgtr 0 .
$$

In our calculation we use first of all solutions of the more general form:

$$
\begin{aligned}
f_{0} & =f_{0}\left(v_{\|}^{2}, v_{\perp}^{2}, p_{y}\right), \\
p_{y} & =v_{y}+\int_{0}^{x} \Omega(\xi) \mathrm{d} \xi ; \quad \Omega=e B / m c .
\end{aligned}
$$

As in Ref. 1, however, we shall confine the discussion of stability to solutions of the more particular form:

$$
f_{0}=f(W, \mu),
$$

where $W=$ kinetic energy, $\mu=$ magnetic moment. Rosenbluth and Krall ${ }^{1}$ may, in our opinion, be simplifying matters at this point by taking

$$
W=v_{\|}^{2}+v_{\perp}^{2} ; \quad \mu=\frac{v_{\perp}^{2}}{B(x)}
$$

with $x=$ coordinate of the particle position. With this definition the equation $f_{0}=f$ cannot be satisfied. The expression for $\mu$ is in fact inexact; according to Northrop ${ }^{3}$, to the first order in the gyroradius and in $\varepsilon \equiv \mathrm{d} \ln B / \mathrm{d} x$ it is

$$
\mu=\frac{v_{\perp}^{\bar{\Sigma}}}{B\left(x_{0}\right)} \approx \frac{v_{\perp}^{2}}{B(0)}\left[1-\frac{\varepsilon}{\Omega(0)}\left(v_{y}+\Omega(0) x\right)\right] .
$$

Here $x_{0}$ ist the $x$-coordinate of the guiding centre of a gyrating particle.

3 T. G. Northrop, The Adiabatic Motion of Charged Particles, Interscience Publishers 1963, p. 69.

${ }^{4}$ N. A. Krall and M. N. Rosenbluth, Phys. Fluids 8, 1488 [1965].
It should be noted that in the zeroth order in $\varepsilon$ it holds that

$$
p_{y}=v_{y}+\Omega(0) x .
$$

Accordingly, to the first order in $\varepsilon$ the magnetic moment $\mu$ is given uniquely by $v_{\perp}^{2}$ and $p_{y}$, and thus the equation $f_{0}=f$ can be satisfied as well.

\section{Perturbations}

As in Ref. 1, the perturbations studied are lowfrequency electrostatic waves, i. e. $\boldsymbol{E}_{1}=-\operatorname{grad} \Phi_{1}$, where $\boldsymbol{E}_{1}$ is the electric field of the perturbation, and

$$
\Phi_{1}=\varphi(x) e^{i \omega t} e^{i k y} e^{i K z} ;
$$

here $\omega$ is complex, $k$ and $K$ are real, and we assume

$$
|\omega| \ll|\Omega| ;\left|k\left\langle v_{\mathrm{D}}\right\rangle\right| \ll|\Omega| ;\left|K\left\langle\left|v_{\|}\right|\right\rangle\right| \ll|\Omega| ;
$$

and $\mathrm{d} \varphi / \mathrm{d} x \rightarrow 0$. Here $\left\langle v_{\mathrm{D}}\right\rangle$ is the mean drift velocity:

$$
\left\langle v_{\mathrm{D}}\right\rangle=\frac{\varepsilon}{2 \Omega_{0}}\left\langle v_{\perp}^{2}\right\rangle
$$

with $\Omega_{0}=$ cyclotron frequency at the location of the guiding centre. Various authors ${ }^{4,5}$ have shown that such perturbations may occur as instabilities ("universal instabilities") in the limiting case $\beta \ll 1$.

With the method of characteristics there then follows from the linearized Vuasov equation,

$\frac{\partial f_{1}}{\partial t}+\boldsymbol{v} \cdot \frac{\partial f_{1}}{\partial \boldsymbol{x}}+\frac{e}{m c}(\boldsymbol{v} \times \boldsymbol{B}) \cdot \frac{\partial f_{1}}{\partial \boldsymbol{v}}=-\frac{e}{m} \boldsymbol{E}_{1} \cdot \frac{\partial f_{0}}{\partial \boldsymbol{v}}$,

and from the Porsson equation,

$$
\operatorname{div} \boldsymbol{E}_{1}=\sum 4 \pi e \iiint f_{1}(\boldsymbol{x}, \boldsymbol{v}) \mathrm{d}^{3} v,
$$

a dispersion relation for the perturbation $\left(f_{1}, \boldsymbol{E}_{1}\right)$ at $x=0$ which has the following general form:

$$
D(\omega, k, K, \ldots)=k^{2}+K^{2} .
$$

\section{Dispersion Relation}

In principle, the exact form of the dispersion relation is derived in the same way as in Ref. 1 , but with allowance for first-order terms in $\varepsilon \equiv \mathrm{d} \ln B / \mathrm{d} x$. In particular, the more exact expression for the magnetic moment (cf. Section 2) is used. Only the most important intermediate results are noted.

5 A. A. Rukhadze and V. P. Silin, Sov. Phys. Uspekhi 7, 209 [1964]. 
By using the method of characteristics and the constants of motion of the equilibrium, $v_{\|}^{2}, v_{\perp}^{2}, p_{y}$, we obtain first of all, as in Ref. 1, the following expression for the function $D$ :

$\begin{aligned} & D=\sum \frac{8 \pi e^{2}}{m}\left\{\iiint \mathrm{d}^{3} v\left[\frac{\partial f_{0}}{\partial v_{\perp}^{2}}\right.\right.-i H(\boldsymbol{v}) \int_{-\infty}^{0} \mathrm{~d} t \\ &\left.\left.\cdot e^{i k\left(y^{\prime}-y\right)} e^{i K\left(z^{\prime}-z\right)} e^{i \omega t}\right]\right\}\end{aligned}$

with

$H(\boldsymbol{v})=\omega \frac{\partial f_{0}}{\partial v_{\perp}^{2}}-K v_{\|}\left(\frac{\partial f_{0}}{\partial v{ }^{2}}-\frac{\partial f_{0}}{\partial v_{\perp}^{2}}\right)-\frac{k}{2 \Omega(0)} \frac{\partial f_{0}}{\partial x}$.

The functions $y^{\prime}(t)$ and $z^{\prime}(t)$ are the $y$ and $z$ coordinates of the particle trajectories in the unperturbed equilibrium configuration, with the "initial" conditions

$y^{\prime}(0)=y ; \quad z^{\prime}(0)=z ; \quad \dot{y}^{\prime}(0)=v_{y} ; \quad \dot{z}^{\prime}(0)=v_{\|}$.

We use the approximate trajectories

$$
\begin{aligned}
x^{\prime} & =x_{0}-\frac{v_{\perp}}{\Omega_{0}} \sin \left(\vartheta-\Omega_{0} t\right), \\
y^{\prime} & =y_{0}(t)+\frac{v_{\perp}}{\Omega_{0}} \cos \left(\vartheta-\Omega_{0} t\right), \\
z^{\prime} & =z+v_{\text {॥ }} t, \\
y_{0}(t) & =y+v_{\mathrm{D}} t-\frac{v_{\perp}}{\Omega_{0}} \cos \vartheta .
\end{aligned}
$$

Here $\Omega_{0}$ is the value of the cyclotron frequency at the location $\left(x_{0}, y_{0}, z\right)$ of the guiding centre of a particle; and

$$
v_{\mathrm{D}}=\varepsilon v_{\perp}^{2} / 2 \Omega_{0}
$$

is the drift velocity perpendicular to the field direction which is caused by the field inhomogeneity.

The trajectories are correct to the first order in $\varepsilon \equiv \mathrm{d} \ln B / \mathrm{d} x$ with the exception of terms containing harmonics of the cyclotron frequency, i. e. all terms of the form

$$
\left[a_{n} \sin \left(n \Omega_{0} t\right)+b_{n} \cos \left(n \Omega_{0} t\right)\right]
$$

with $n \geqq 2$ were omitted in the expressions for $x^{\prime}$ and $y^{\prime}$.

In the expression for the function $D$, the $t$-integration is carried out by using the equation

$$
e^{i p \sin q}=\sum_{n=-\infty}^{+\infty} e^{i n q} J_{n}(p) .
$$

Allowing for $|\omega|,\left|K\left\langle\left|v_{11}\right|\right\rangle\right|,\left|k\left\langle v_{\mathrm{D}}\right\rangle\right|$ all $\ll|\Omega|$ one obtains:

$$
\begin{array}{r}
D=\sum \frac{8 \pi e^{2}}{m} \iiint \mathrm{d}^{3} v\left[\frac{\partial f_{0}}{\partial v_{\perp}^{2}}-\frac{H(v) J_{0}\left(k v_{\perp} / \Omega_{0}\right)}{\omega+K v_{11}+k v_{\mathrm{D}}}\right. \\
\left.\cdot \sum_{n=-\infty}^{+\infty} e^{i n\left(\vartheta+\frac{1}{2} \pi\right)} J_{n}\left(-\frac{k v \perp}{\Omega_{0}}\right)\right] .
\end{array}
$$

We are interested in $f_{1}$ as an asymptotic solution of an initial value problem in time; consequently, the $\boldsymbol{v}$-integration must be carried out with $\operatorname{Im}[\omega]<0$, this being equivalent to unstable solutions. The dispersion relation for stable solutions. i. e. for $\operatorname{Im}[\omega] \geqq 0$ would follow in the usual way by continuing $D$ analytically into the upper $\omega$ half-plane.

Next we integrate over $\vartheta$ in the expression for $D$. For this purpose the dependence of the integrand on $\vartheta$ has to be known. The angle $\vartheta$ is defined in the $\boldsymbol{v}$. space by the initial conditions for the equations of the trajectories:

$$
\begin{aligned}
& v_{x}=v_{\perp} \cos \vartheta \\
& v_{y}=v_{\perp} \sin \vartheta+v_{\mathrm{D}} ; \\
& v_{\mathrm{D}}=\frac{\varepsilon v_{\perp}^{2}}{2 \Omega_{0}} ; \quad \Omega_{0}=\Omega_{0}(\boldsymbol{v}) .
\end{aligned}
$$

The values $\boldsymbol{v}, \Omega_{\mathbf{0}}, v_{D}$ may be expressed by $\imath_{\|}, v_{\perp}$, $\vartheta$, and $\Omega(0)$. The dependence of the integrand on $\vartheta$ is found by expanding in powers of $\varepsilon \equiv \mathrm{d} \ln B / \mathrm{d} x$ and taking into account all terms of the zeroth or first orders in $\varepsilon$. The more particular form $f_{0}=f(W, \mu)$ of the equilibrium distribution functions is here utilized for the first time. The magnetic moment $\mu$ is also expanded in powers of $\varepsilon$ :

$$
\begin{aligned}
& \mu=\mu_{0}+\varepsilon \mu_{1} ; \mu_{0} \equiv v_{\perp}^{2} / B(0) ; \\
& \mu_{1} \equiv-\frac{v_{\perp}^{2}}{B(0) \Omega(0)}\left(v_{\perp} \sin \vartheta+\Omega(0) x\right) .
\end{aligned}
$$

When the integration over $\vartheta$ has been carried out and all terms of the second order upwards in $\varepsilon$ are dropped the dispersion relation can be given the following form:

$$
\begin{gathered}
D(\omega, k, K, \ldots)=k^{2}+K^{2} ; \\
D \equiv D_{1}(k)+D_{2}(\omega, k, K) ; \\
D_{1} \equiv \sum \frac{16 \pi^{2} e^{2}}{m} \int_{0}^{\infty} v_{\perp} \mathrm{d} v_{\perp} \int_{-\infty}^{+\infty} \mathrm{d} v_{\|} \\
\cdot\left\{\frac{\partial f}{\partial W}+\frac{1}{B(0)} \frac{\partial f}{\partial \mu}\left[1-J_{0}^{2}\left(\frac{k v \perp}{\Omega(0)}\right)\right]\right\} ; \\
D_{2} \equiv \omega \int_{-\infty}^{+\infty} \mathrm{d} u \frac{P(u, k, K)}{\omega+K u} ; \\
P \equiv-\left.\sum \frac{16 \pi^{2} e^{2}}{m} \int_{0}^{\infty} v_{\perp} \mathrm{d} v_{\perp} \frac{\partial f}{\partial W}\right|_{u} J_{0}^{2}\left(\frac{k v \perp}{\Omega(0)}\right) .
\end{gathered}
$$

The derivatives of $f$ should be taken here at $\left(W, \mu_{0}\right)$, so that $p_{y}=0$, not at $(W, \mu)$. The notation $\left.\right|_{u}$ means that the particular value

$$
v_{\text {॥ }}=u-k \varepsilon v^{2} / 2 K \Omega(0)
$$

should be substituted. 
The dispersion relation obtained is identical with that given in Ref. 1 because all first-order correction terms in $\varepsilon \equiv \mathrm{d} \ln B / \mathrm{d} x$ cancel one another out.

\section{Properties of the Nyquist Diagram}

In the following we use the term "Nyquist diagram" to refer specifically to the mapping of the real $\omega$-axis $\left(\omega=\omega_{1}\right)$, traversed in the positive direction, into the complex $D$-plane by means of the function $\mathrm{D}(\omega, k, K, \ldots)$. For the purpose of discussion we use the equations following from Section 4:

$$
\begin{aligned}
& \operatorname{Im}\left[D\left(\omega_{1}, k, K\right)\right]=- \\
& \left.\frac{16 \pi^{3} \omega_{1}}{|K|} \sum \frac{e^{2}}{m} \int_{0}^{\infty} v_{\perp} \mathrm{d} v_{\perp} J_{0}^{2}\left(\frac{k v_{\perp}}{\Omega}\right) \frac{\partial f}{\partial W}\right|_{\text {res }} ; \\
& D(0)=\sum \frac{16 \pi^{2} e^{2}}{m} \int_{0}^{\infty} v_{\perp} \mathrm{d} v_{\perp} \int_{-\infty}^{+\infty} \mathrm{d} v_{\|} \\
& \cdot\left\{\frac{\partial f}{\partial W}+\frac{1}{B} \frac{\partial f}{\partial \mu}\left[1-J_{0}^{2}\left(\frac{k v_{\perp}}{\Omega}\right)\right]\right\}=\mathrm{real} ; \\
& D(\infty)=\sum \frac{16 \pi^{2} e^{2}}{m} \int_{0}^{\infty} v_{\perp} \mathrm{d} v_{\perp} \int_{-\infty}^{+\infty} \mathrm{d} v_{\mathrm{ii}} \\
& \cdot\left(\frac{\partial f}{\partial W}+\frac{1}{B} \frac{\partial f}{\partial \mu}\right)\left[1-J_{0}^{2}\left(\frac{k v_{\perp}}{\Omega}\right)\right]=\mathrm{real} ; \\
& D(\infty)-D(0)=\int_{0}^{\infty} \int_{\perp}^{\infty} v_{\perp} \mathrm{d} v_{\perp}^{+\infty} \mathrm{d} v_{\|} \frac{\partial f}{\partial W} J_{0}^{2}\left(\frac{k v_{\perp}}{\Omega}\right) .
\end{aligned}
$$

Here the notation $\left.\right|_{\text {res }}$ means that

$$
v_{\text {" res }} \equiv-\left(\omega_{1}+k v_{\mathrm{D}}\right) / K
$$

should be substituted for $v_{\| 1}$. Again the derivatives of $f$ should be taken at $\left(W, \mu_{0}\right)$, i. e. $p_{y}=0$.

With the exception of the special cases dealt with in Section 10, the following is true: If $f$ tends sufficiently strongly towards zero for large velocities the Nyquist diagram is a closed curve. If, in addition, $\partial f / \partial W<0$ there are only two zeros of $\operatorname{Im}\left[D\left(\omega_{1}\right)\right]$, namely for $\omega_{1}=0$ and $\omega_{1}= \pm \infty$. The Nyquist diagram thus intersects the real $D$ axis only at the two points $D(0)$ and $D(\infty), D(0)$ being smaller than $D(\infty)$ except in a few special cases (cf Section 10). See Fig. 1.

The values $D(0)$ and $D(\infty)$ depend neither on $K$ nor $\varepsilon$. If $k^{2}<D(\infty)$ is valid for a particular $k$ it is

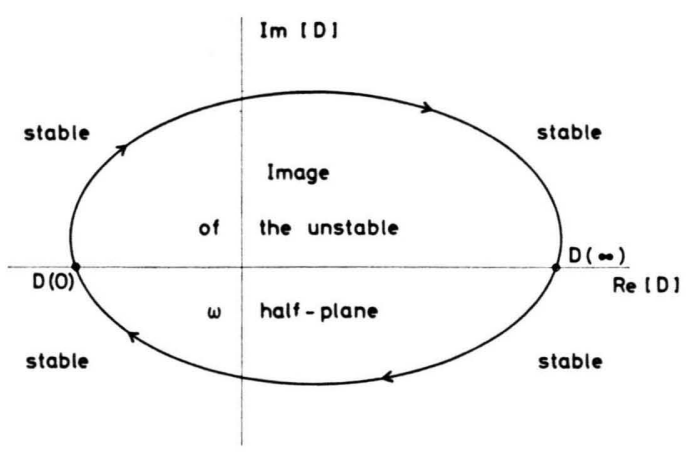

Fig. 1. Nyquist diagram for $\partial f / \partial W<0$, schematic.

possible to determine a $K$ for every real $D=D_{r}$ with $k^{2}<D_{\mathrm{r}}<D(\infty), D(0)<\mathrm{D}_{\mathrm{r}}$, so that the dispersion relation $D_{\mathrm{r}}=k^{2}+K^{2}$ is satisfied, and from inversion of the conformal mapping $D(\omega)$ we obtain the corresponding frequency $\omega\left(D_{\mathrm{r}}\right)$ located in the unstable $\omega$ half-plane, i.e. with $\operatorname{Im}[\omega]<0$. The fact that $D(\omega)$ is free of singularities in the unstable $\omega$ halfplane can easily be proved for a fairly general class of distribution functions; cf Ref. 6 for example. For the dispersion relation with $\partial f / \partial W<0$ the following theorems are thus valid:

Theorem 1: The modes with $k=\mathrm{k}_{\mathbf{0}}$ are all stable if, and only if, $D\left(\omega=\infty, k_{0}, \ldots\right) \leqq k_{0}{ }^{2}$ is valid.

Theorem 2: For stability it is necessary and sufficient that $D(\omega=\infty, k, \ldots) \leqq k^{2}$ for all real $k$. In particular, $D(\infty) \leqq 0$ is sufficient for stability.

It would thus be sufficient for stability is (cf Section 6) not only

$$
\frac{\partial \tilde{f}_{0}}{\partial v \|^{2}} \equiv \frac{\partial f}{\partial W}<0
$$

were valid, but also

$$
\frac{\partial \tilde{f}_{0}}{\partial v_{\perp}^{2}} \equiv \frac{\partial f}{\partial W}+\frac{1}{B} \frac{\partial f}{\partial \mu}<0 .
$$

The latter condition cannot be satisfied identically in a minimum- $B$ field because of the existence of the loss cone in the velocity space, but it can be in an infinitely extended magnetic field constant along the lines of force. For $f_{0}=f(W, \mu), \partial f / \partial W<0$, a nonmonotonic $v_{\perp}$-distribution is thus necessary for electrostatic drift instabilities to occur. These are thus loss-cone unstable drift waves.

6 R. Balescu, Statistical Mechanics of Charged Particles, Interscience Publishers 1963, Appendix 2. 


\section{Example of an Unstable Equilibrium}

We shall give now an example of an unstable equilibrium with distribution functions of the form

$$
f_{0}=f(W, \mu), \frac{\partial f}{\partial W}<0,
$$

in keeping with the TAylor condition ${ }^{2}$. From Sec-tion 4 it follows that the transformation equations

$$
\begin{gathered}
W=v_{\|}^{2}+v_{\perp}^{2} ; \quad \mu=\mu_{0} \equiv v_{\perp}^{2} / B(0) ; \\
\frac{\partial f}{\partial W}=\frac{\partial \tilde{f}_{0}}{\partial v^{2}} ; \quad \frac{1}{B(0)} \frac{\partial f}{\partial \mu}=\frac{\partial \tilde{f}_{0}}{\partial v_{\perp}^{2}}-\frac{\partial \tilde{f}_{0}}{\partial v_{\|}^{2}} ; \\
\tilde{f}_{0}\left(v_{\|}^{2}, v_{\perp}^{2}\right) \equiv f_{0}\left(v_{\|}^{2}, v_{\perp}^{2}, p_{y}=0\right)
\end{gathered}
$$

have to be used in the evaluation of the dispersion relation. It is therefore sufficient to study any $\tilde{f}_{0}\left(v_{\|}^{2}, v_{\perp}^{2}\right)$ with $\partial \tilde{f}_{0} / \partial v_{\|}^{2}<0$.

We define

$$
F_{0}\left(v_{\perp}\right) \equiv \frac{1}{N_{0}} \int_{-\infty}^{+\infty} \tilde{f_{0}} \mathrm{~d} v_{\text {। }}
$$

with the normalization

$$
2 \pi \int_{0}^{\infty} F_{0}\left(v_{\perp}\right) v_{\perp} \mathrm{d} v_{\perp}=1 .
$$

On integration by parts we obtain:

$$
\begin{aligned}
D(\infty)=\sum \frac{\omega_{\mathrm{p}}{ }^{2}|k|}{|\Omega|} \int_{0}^{\infty} 2 \pi v_{\perp} \mathrm{d} v_{\perp} \\
\cdot F_{0}\left(v_{\perp}\right) \frac{J_{0}{ }^{2}(|k v \perp / \Omega|)}{v_{\perp}}
\end{aligned}
$$

with

$J_{0}{ }^{\prime}(x) \equiv \frac{\mathrm{d} J_{0}{ }^{2}}{\mathrm{~d} x} ; J_{0}=$ zeroth-order BESSEL function.

Let us now take in particular

so that

$$
F_{0}\left(v_{\perp}\right)=\frac{1}{2 \pi V_{\perp}} \delta\left(v_{\perp}-V_{\perp}\right)
$$

$$
D(\infty)=\sum \frac{\omega_{\mathrm{p}}{ }^{2}|k|}{|\Omega| V_{\perp}} J_{0}{ }^{2}\left(\frac{|k| V_{\perp}}{|\Omega|}\right) .
$$

For $x=n \pi, n \gg 1$, it holds that

$$
J_{0}{ }^{\prime \prime}(x=n \pi) \sim \frac{2}{\pi x}\left(1-\frac{1}{x}\right)>\frac{1}{\pi x} .
$$

Accordingly, we obtain for $|k| V_{\perp}=n \pi|\Omega|, n \gg 1$, with $\sqrt{\pi / 2}|k| V_{\perp}<\omega_{\mathrm{p}}$ :

$$
D(\infty)>\frac{1}{\pi} \sum \frac{\omega_{\mathrm{p}}^{2}}{V_{\perp}}>k^{2},
$$

i. e. instability (according to Theorem 2 of Section $5)$ for plasma with $\left|\Omega_{\mathrm{e}}\right| \ll \omega_{\text {pe }}$ although $\partial f / \partial W<0$; or, as the derivation shows, irrespective of whether $\partial f / \partial W<0$ is satisfied identically or not, provided the properties of the Nyquist diagram that are used are the same as in the case $\partial f / \partial W<0$.

As can readily be shown, instability is obtained in the same way in the case $\left|\Omega_{\mathrm{e}}\right| \gg \omega_{\mathrm{pe}}$, $\left|\Omega_{\mathrm{i}}\right| \ll \omega_{\mathrm{pi}}$, this being due to the ion contribution to the dispersion relation (cf Section 9). In order for the instabilities to be physically real, it is necessary that $|\omega| \ll|\Omega|$, among other conditions. There can be unstable solutions with arbitrarily small $|\omega|$ if $k^{2} \approx D(0)>0$. In the special case under consideration it can be shown that $k^{2} \approx D(0)>0$ can be satisfied if, for instance, $T_{\mathrm{i} \perp}=T_{\mathrm{e} \perp}, T_{\mathrm{i}_{\mathrm{i}}}=T_{\mathrm{e} \|}$, $2 \omega_{\mathrm{pi}}^{2}>n_{\mathrm{i}}^{2} \pi^{3} \Omega_{\mathrm{i}}^{2}$, and if $V_{\perp}^{2}$ is not very much smaller than $V_{\|}^{2}$, where $V_{\|}$is a suitable mean value of $\left|v_{\|}\right|$. A more exact study is made only in the spatially homogeneous case.

\section{The Spatially Homogeneous Case}

As stated, the expressions for $D(0)$ and $D(\infty)$ do not depend on $\varepsilon$. The instabilities thus persist for $\varepsilon \rightarrow 0$ (spatially homogeneous case) as pure losscone instabilities. These are not identical, however, with the high-frequency loss-cone instabilities found by Rosenbluth and Post ${ }^{7}$ because if $\omega_{2} \equiv \operatorname{Im}[\omega]<0$, it holds that

$$
\begin{array}{r}
\operatorname{Im}[D]= \\
=i \omega_{2} \sum 4 \pi \omega_{\mathrm{p}}^{2} \int_{0}^{\infty} v_{\perp} \mathrm{d} v_{\perp} \int_{0}^{\infty} \mathrm{d} v_{\|} \frac{\partial f}{\partial W} J_{0}^{2}\left(\frac{k v \perp}{\Omega}\right) \\
\quad \cdot \frac{4 \omega_{1}\left(K v_{\|}\right)^{2}}{\left[\left(\omega_{1}+K v_{\|}\right)^{2}+\omega_{2}^{2}\right]\left[\left(\omega_{1}-K v_{\|}\right)^{2}+\omega_{2}^{2}\right]} .
\end{array}
$$

Thus, only for $\omega_{1} \equiv \operatorname{Re}[\omega]=0$ does one obtain $\operatorname{Im}[D]=0$, i.e. unstable solutions. We now show that, under special conditions at any rate, there are unstable solutions which satisfy the inequalities $|\omega| \ll|\Omega|$ and $|K<| v_{n}|>| \ll|\Omega|$. For $\varepsilon=0$ and the special case of Section 6 the dispersion relation is

$$
k^{2}+K^{2}=\operatorname{Re}[D]_{\omega_{1}=0}
$$

or

$$
k^{2}+K^{2} \sim \frac{2}{\pi} \sum \frac{\omega_{\mathrm{p}}^{2}}{V_{\perp}^{2}}\left[1-\frac{1}{2} \frac{|\Omega|}{|k| V_{\perp}} \frac{\left(K V_{\perp}\right)^{2}}{\omega_{2}^{2}}\right]
$$

7 M. N. Rosenbluth and R.F.Post, Phys. Fluids 8, 547 [1965]. 
with $V_{\perp}=n \pi|\Omega| /|k|, n_{\mathrm{i}, \mathrm{e}} \gg 1$, and $|K| V_{\|} \ll\left|\omega_{2}\right|$, where $V_{\|}$is a suitable mean value of $\left|v_{\|}\right|$which, however, no longer occurs in the approximate dispersion relation. Inserting $V_{\perp}$ and solving for $\omega_{2}{ }^{2}$ gives

$$
\omega_{2}^{2} \sim \frac{K^{2}}{k^{2}} \frac{\sum \omega_{\mathrm{p}}^{2} / n \pi}{\sum\left(2 \omega_{\mathrm{p}}^{2} / n^{2} \pi^{2} \Omega^{2}\right)-\left(1+K^{2} / k^{2}\right)} .
$$

In the case $T_{\mathrm{e} \perp}=T_{\mathrm{i} \perp}$ it holds that $n_{\mathrm{e}} / n_{\mathrm{i}}=\sqrt{m_{\mathrm{e}} / m_{\mathrm{i}}}$, and for $K^{2} \ll k^{2}, \omega_{\mathrm{pi}} \geqq n_{\mathrm{i}} \pi|\Omega|$, it follows that

$$
\omega_{2}^{2} \lesssim \pi \frac{K^{2}}{k^{2}}\left(\frac{m_{\mathrm{i}}}{m_{\mathrm{e}}}\right)^{3 / 2} n_{\mathrm{i}} \Omega_{\mathrm{i}}{ }^{2} .
$$

If $K$ is chosen sufficiently small, we have $\left|\omega_{2}\right| \ll\left|\Omega_{\mathrm{i}}\right|$. Because of $|K| V_{\|} \ll\left|\omega_{2}\right|$ it then holds as well that $|K| V_{\text {!! }} \ll|\Omega|$.

\section{Critique of the Stability Criterion of Rosenbluth and Krall}

We have given an example proving that the TAylor condition ${ }^{2}, f_{0}=f(W, \mu)$ with $\partial f / \partial W<0$, is not sufficient for stability with respect to the dispersion relation of Section 4. This, in turn, proves that the condition used by Rosenbluth and Krall ${ }^{1}$ as a sufficient stability criterion,

$$
\begin{gathered}
\langle\boldsymbol{j} \cdot \boldsymbol{E}\rangle \propto \omega_{1} \operatorname{Im}\left[D\left(\omega_{1}, k, K, \ldots\right)\right] \geqq 0 \\
\text { for }
\end{gathered}
$$

is not a valid criterion in the case under investigation. The criterion is actually satisfied for distribution functions $f_{0}=f(W, \mu)$ with $\partial f / \partial W<0$ by the function $D$ given in Section 4 . Since, however, according to Section 6 instabilities still occur if the equilibrium is chosen properly the criterion of Rosenbluth and $\mathrm{KRALL}^{1}$ is clearly not valid in the present case.

\section{Stable Equilibria}

In Section 6 it is demonstrated that there are unstable equilibria with $\left|\Omega_{\mathrm{i}}\right| \ll \omega_{\mathrm{pi}}$ and with distribution functions of the form $f_{0}=f(W, \mu), \partial f / \partial W<0$, and

$$
F_{0}\left(v_{\perp}\right)=\frac{1}{2 \pi V_{\perp}} \delta\left(v_{\perp}-V_{\perp}\right) .
$$

We shall now show that the same distribution function is stable for $\left|\Omega_{\mathrm{i}}\right| \gg \omega_{\mathrm{pi}}$. For this purpose we again consider

$$
D(\infty)=\sum \frac{\omega_{\mathrm{p}}{ }^{2}|k|}{|\Omega| V_{\perp}} J_{0}{ }^{2}\left(\frac{|k| V_{\perp}}{|\Omega|}\right)
$$

and show that $D(\infty) \ll k^{2}$ for $|k| V_{\perp} \ll|\Omega|$, $|k| V_{\perp} \approx|\Omega|$, and $|k| V_{\perp} \gg|\Omega|$. According to Theorem 2 in Section 5 this is sufficient for stability. In our proof we make use of the fact that the inequality $\left|\Omega_{\mathrm{e}}\right| \gg \omega_{\mathrm{pe}}$ follows from $\left|\Omega_{\mathrm{i}}\right| \gg \omega_{\mathrm{pi}}$.

The following relations are satisfied in order of magnitude:

$$
\begin{aligned}
& J_{0}{ }^{\prime \prime} \approx=\frac{|k| V_{\perp}}{|\Omega|} \text { for }|k| V_{\perp} \ll|\Omega| ; \\
& J_{0}{ }^{2} \lesssim 1 \text { for }|k| V_{\perp} \approx|\Omega| ; \\
& J_{0}{ }^{{ }^{\prime}}<\frac{4}{\pi} \frac{|\Omega|}{|k| V_{\perp}} \text { for }|k| V_{\perp} \gg|\Omega| .
\end{aligned}
$$

Substituting the values, we obtain, for the ion and electron contributions to $D(\infty)$ :

$$
\begin{aligned}
& D_{\mathrm{i}, \mathrm{e}}(\infty) \approx-\frac{\omega_{\mathrm{pie \textrm {e }}}^{2} k^{2}}{\Omega_{\mathrm{i}, \mathrm{e}}^{2}}<0 \text { for }|k| V_{\perp} \ll|\Omega| ; \\
& D_{\mathrm{i}, \mathrm{e}}(\infty) \underset{\approx}{\frac{\omega_{\mathrm{p}, \mathrm{e}}^{2}|k|}{\left|\Omega_{\mathrm{i}, \mathrm{e}}\right| V_{\perp \mathrm{i}, \mathrm{e}}}} \approx \frac{\omega_{\mathrm{ple}, k^{2}}^{2}}{\Omega_{\mathrm{i}, \mathrm{e}}^{2}} \\
& \text { for }|k| V_{\perp} \approx|\Omega| \text {; } \\
& D_{\mathrm{i}, \mathrm{e}}(\infty)<\frac{4}{\pi} \frac{\omega_{\mathrm{pi, \textrm {e }}}^{2}}{V_{\perp 1, \mathrm{e}}^{2}} \ll \frac{4}{\pi} \frac{\omega_{\mathrm{p}, \mathrm{e}}^{2} k^{2}}{\Omega_{1, \mathrm{e}}^{2}} \\
& \text { for }|k| V_{\perp} \gg|\Omega| \text {. }
\end{aligned}
$$

Thus, for $|\Omega| \gg \omega_{\mathrm{p}}$ stability does in fact follow, and this irrespective of the choice of $V_{\perp}$. From this it follows that in the case $\left|\Omega_{\mathrm{i}}\right| \gg \omega_{\mathrm{pi}}$ every superposition of distribution functions of the form in question, i. e. every $f_{0}=f(W, \mu)$ whatsoever with $\partial f / \partial W<0$, is also stable with respect to the instabilities under consideration. The condition $\left|\Omega_{\mathrm{i}}\right| \gg \omega_{\mathrm{pi}}$ is satisfied only in the case of plasma of relatively low density in a magnetic field; for example, for $B=10^{4}$ gauss it follows that the plasma density has to be much less than $5 \times 10^{9} \mathrm{~cm}^{-3}$.

\section{Singular Cases}

The statements made in Section 5 are not true in a few singular cases.

a) Case $K=0, \varepsilon \neq 0, k \neq 0$ :

The Nyquist diagram becomes singular since part of the boundary curve coindides with part of the real $D$-axis. Since, however, $\operatorname{Im}[D] \neq 0$ for $\operatorname{Im}[\omega]<0$ there are no unstable solutions.

b) Case $K=\varepsilon=0, k \neq 0$ :

The Nyquist diagram degenerates to a point; $D(\omega) \equiv D(\infty)$ for $\operatorname{Im}[\omega]<0$. There is a degenerate set of unstable solutions with arbitrary $\omega, \operatorname{Im}[\omega]<0$, for $k^{2}=k_{0}^{2} \equiv D(\infty)$, while for 
$k^{2} \neq k_{0}{ }^{2}$ no solution exists. These solutions are probably not physically meaningful since it is to be expected that no degeneracy occurs in the exact dispersion relation.

c) For special equilibrium distribution as well, the Nyquist diagram may degenerate to a point, $D(\omega) \equiv D(\infty)$, namely for

$$
\tilde{f}_{0}=\frac{1}{2 \pi V_{\perp}} \delta\left(v_{\perp}-V_{\perp}\right) g_{\perp}\left(v_{\perp}\right) g_{\|}\left(v_{\|}\right)
$$

with

$$
\begin{gathered}
g_{\perp}\left(V_{\perp}\right)=1 ; \int_{-\infty}^{+\infty} g_{\|} \mathrm{d} v_{\|}=1 ; \\
J_{0}\left(\frac{k V_{\perp}}{\Omega}\right)=0 ;
\end{gathered}
$$

and for normalized sums or infinite series os such $\tilde{f_{0}}$. The degeneracy is eliminated, however, when the $\delta$-function is approximated by a smooth function, and so this case has no physical significance.

It is perhaps not superfluous to mention that the special case studied in Sections 6, 7, 9 do not represent non-physical limiting cases, although there, too, the equilibrium distributions are represented for simplicity by means of $\delta$-functions. However, $J_{0}{ }^{\prime}\left(k V_{\perp} / \Omega\right)$ and $J_{0}\left(k V_{\perp} / \Omega\right)$ are not equal to zero, and so there are continuous approximations of the $\delta$-function for which the results obtained are correct in arbitrarily good approximation.

\section{Conclusion}

The foregoing results may be summarized as follows:

a) The dispersion relation of Rosenbluth and $\mathrm{KRALL}{ }^{1}$ is correct to first order in the gyroradii and in $\varepsilon \equiv \mathrm{d} \ln B / \mathrm{d} x$ within the limits of the other approximations used. b) The stability criterion used by Rosenbluth and $\mathrm{KRALL}^{1},\langle\boldsymbol{j} \cdot \boldsymbol{E}\rangle \geqq 0$ for $\omega$ real, may cease to have validity if approximate expressions for the dispersion relation are used. In the present case this is in fact so since a low-frequency approximation is used with $D(\infty)$ generally non-vanishing. The stability criterion in question would be valid for $D(0)<D(\infty) \leqq 0$, as may be deduced from the Nyquist diagram.

c) The stabilization theorem of Rosenbluth and $\mathrm{K}_{\mathrm{RALL}}{ }^{1}$ is not valid. Satisfying the Taylor condition $^{2}$ alone is generally not sufficient for stability with respect to electrostatic drift instabilities. When the TAYLOR condition is satisfied, however, the presence of a non-monotonic $v_{\perp}$-distribution is necessary before electrostatic drift instabilities will occur. With the exception of a few simple special cases numerical calculations will be needed in order to decide whether a particular minimum- $B$ equilibrium is stable with respect to electrostatic drift instabilities.

d) The existence of new types of instabilities is proven. These are a new type of loss cone instability, with $\operatorname{Re}[\omega]=0$, in a homogeneous magnetic field and loss-cone unstable drift waves in an inhomogeneous magnetic field. The proof was carried out for equilibria satisfying the TAYLOR condition ${ }^{2}$, $f_{0}=f(W, \mu), \partial f / \partial W<0$; it is conceivable, how. ever, that these instabilities occur in other cases as well.

\section{Acknowledgment}

The authors wish to express their appreciation to Dr. H. TAsso and Dr. R. Gorenflo for several interesting discussions. 